\title{
UN'ANALISI ISTITUZIONALE DEL DECLINO DELL'ECONOMIA ITALIANA
}

\author{
ANDREA CAPUSSELA $(*)$ \\ Nota presentata dal m.e. Renata Targetti Lenti \\ (Adunanza del 14 novembre 2019)
}

SUNTO. - Questo saggio tenta un'interpretazione del recente declino economico dell'Italia, che iniziò circa due decenni prima della recessione provocata dalla crisi finanziaria globale del 2007-8. La tesi è che le radici profonde del declino risiedono nel terreno dell'economia politica della crescita. Questa interpretazione è illustrata tramite un breve esame del percorso di sviluppo dell'Italia e dell'evoluzione del suo equilibrio politico-economico. L'enfasi è posta sulla convergenza del paese alla frontiera mondiale della produttività, sulle sue prestazioni in termini di produttività totale dei fattori, e sull'evoluzione del suo ordine sociale e delle sue istituzioni. La lente attraverso la quale queste vicende sono osservate, per gettare luce sulle origini degli attuali vincoli alla crescita, sono la teoria Schumpeteriana della crescita e la teoria economica delle istituzioni. Per illustrare la tesi di fondo, il saggio usa un esempio semplificato dei rapporti tra cittadini e imprese, da un lato, e le autorità pubbliche, dall'altro. All'inadeguata fornitura di servizi pubblici, cittadini e imprese possono reagire in due modi principali: una risposta 'opportunistica' - evadere le imposte, corrompere o accettare relazioni clientelari, al fine di procurarsi beni privati - ovvero una risposta 'virtuosa', che fa leva sull'esercizio dei diritti civili e politici per fare valere la responsabilità politica delle autorità pubbliche. Nella prospettiva dei cittadini e delle imprese comuni questo dilemma tipicamente ricalca la struttura del gioco di coordinamento, che ha equilibri molteplici. La razionalità individuale può quindi condurre a una spirale, nella quale più circoli viziosi paralleli trascinano la società su un equilibrio inefficiente caratterizzato da debole responsabilità politica, debole supremazia della legge, e produttività ristagnante.

$* * *$

ABSTRACT. - This paper attempts an interpretation of Italy's recent economic decline, which began two decades before the Great Recession. It argues that its deeper roots lie

(*) Visiting fellow. London School of Economics, London, UK.

E-mail: andrea.capussela@gmail.com 
in the political economy of growth. This interpretation is illustrated through a discussion of Italy's recent growth trajectory and of its politico-economic equilibrium. The emphasis is placed on the country's convergence to the productivity frontier and TFP performance, and on the evolution of its social order and institutions. The lens through which this case is reviewed, to illuminate the origins and evolution of the current constraints to growth, is drawn from institutional economics and Schumpeterian growth theory. It is exemplified by analysing two alternative reactions to the insufficient provision of public goods: an opportunistic one-employing tax evasion, corruption, or clientelism as means to appropriate private goods-and one based on enforcing political accountability. From the perspective of ordinary citizens and firms such social dilemmas can typically be modelled as coordination games, which have multiple equilibria. Self-interested rationality can thus lead to a spiral, in which several mutually reinforcing vicious circles lead society onto an inefficient equilibrium characterized by low political accountability and weak rule of law.

Questo contributo vuole proporre un'analisi delle cause profonde del malessere del paese. Ma prima conviene giustificare l'uso di quel termine, 'declino', che non ha una precisa definizione nella letteratura storica ed economica, ed è carico di suggestioni. La ragione sta nell'evoluzione della produttività totale dei fattori (PTF), che nelle economie avanzate è la principale determinante della crescita di lungo periodo.

\section{IL DECLINO DELL'ECONOMIA ITALIANA}

Dopo sette duri anni, nel corso del 2014 l'economia italiana tornò a crescere. La fine di questa pressoché ininterrotta recessione, la più lunga nell'Eurozona e la più profonda nella storia unitaria dell'Italia in tempo di pace, permise al Fondo monetario internazionale (Fmi) di spostare la propria attenzione dalle conseguenze alle cause (Fmi 2015a, 5-6):

le stime della crescita potenziale, circa $1 / 2$ punto percentuale, sono basse [e] furono drasticamente ridotte nel corso dei passati 8 anni [...]. [L]a crescita potenziale fletté significativamente già [prima del 2008] e calò ulteriormente durante la crisi. La caduta della crescita della PTF spiega gran parte della flessione antecedente la crisi.

È questo il punto di partenza. La principale causa prossima del malessere economico del paese è la flessione della PTF, che «cumulativamente cadde di 7.5 punti percentuali da quando l'Italia adottò l'euro, nel 1998» (Fmi 2015b, 4). 
$\mathrm{Nel}$ confronto con le altre economie industriali avanzate questo crollo è «un'anomalia» (Broadberry et al. 2013, 217). E se è vero che sul finire del 2014 la PTF tornò a crescere, l'accelerazione fu molto più timida che nelle principali economie dell'Eurozona (Fmi 2017, figura 1). Nonostante la profondità della recessione, nel biennio 2015-16 il tasso reale di crescita dell'economia italiana - 0.8\% per anno (Fmi $2017,4)$ - fu circa la metà di quello medio dell'Eurozona, e pare sia stato in larga misura dovuto alla combinazione tra un contesto macroeconomico inusualmente favorevole - grazie a bassi tassi di cambio, tassi d'interesse e prezzi del petrolio, e allo stimolo fornito dall'eccezionale politica monetaria della Bce - e una politica fiscale moderatamente espansiva (Banca d'Italia 2016, 49-52; Fmi 2017), la quale fu possibile, tra l'altro, anche grazie a flessibili interpretazioni delle regole europee. Dunque non sorprende che le più recenti proiezioni del Fmi (2017, 11; 2018) prevedano un ritmo di crescita - circa $1 \%$ per anno in media nel periodo 2018-20 - che, come si è detto, riporterà il PIL al livello registrato nel 2007 solo attorno della metà del prossimo decennio.

Per apprezzare la gravità delle condizioni del paese bisogna però spingere lo sguardo più indietro nel tempo. Il rallentamento della PTF precede infatti non solo la Grande Recessione ma anche l'adozione dell'euro (Broadberry et al. 2013). La - traccia l'evoluzione della PTF in Italia e nei suoi pari in rapporto alla dinamica osservata negli Stati Uniti, l'economia che in questi sette decenni generalmente espresse i più alti livelli di produttività. Le curve del grafico quindi non segnano il livello del progresso tecnologico e organizzativo raggiunto dalle sei economie considerate, ma la loro convergenza verso un obiettivo mobile, che approssimativamente coincide con la frontiera della produttività; e ciò che conviene notare non è solo il percorso dell'Italia, ma anche il rapporto tra la sua traiettoria di convergenza e quella delle cinque economie usate come comparatori.

In Italia la ricostruzione post-bellica fu relativamente rapida. Grazie a una «forte ripresa» della PTF già a partire dal 1945 (Broadberry et al. 2013, 296), nel decennio successivo il paese mosse da un livello di convergenza più elevato dei suoi alleati durante la guerra, Germania e Giappone. Nel ventennio seguente la sua ascesa fu particolarmente rapida: solo la Francia, come l'Italia, raggiunse e poi superò il livello della PTF degli Stati Uniti, sul finire degli anni settanta. Ma il picco toccato dall'Italia fu più alto, nel 1980. Poi ci fu una svolta. Se fino a quell'anno il paese non smise mai di avvicinarsi alla frontiera, 
spesso più velocemente dei suoi pari, da allora iniziò a divergerne, seguito prima dalla Gran Bretagna e poi da altri, in una progressione discendente che accelerò attorno all'inizio del nuovo secolo.

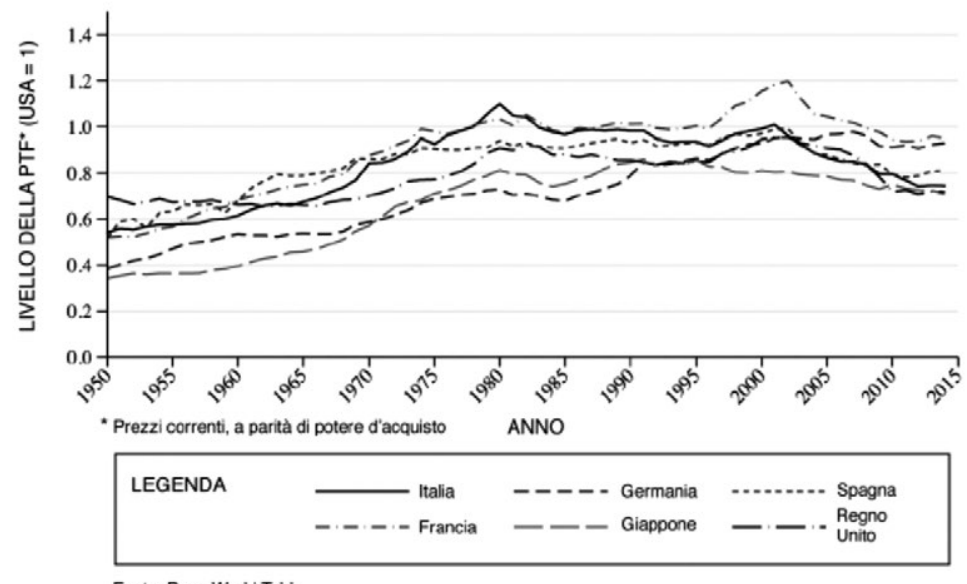

Fonte: Penn World Table

Fig. 1 - Andamento comparato della PTF, 1950-2014.

La Fig. 2 enfatizza questa svolta. Per aumentare il contrasto, le sue curve prendono come riferimento il livello di convergenza sulla PTF degli Stati Uniti - ossia il livello indicato nella Figura 1 - raggiunto da ciascuna economia nell'anno che segnò il culmine dell'ascesa dell'Italia, il 1980, e disegnano l'evoluzione del rapporto tra tale livello e quello osservato quattro decenni successivi. Quindi tutte le curve hanno la medesima origine, e in seguito divergono a seconda di quanto ogni economia abbia migliorato o peggiorato la propria prestazione - in termini di convergenza alla frontiera (mobile) della produttività - rispetto al 1980. I due estremi, pressoché speculari, sono la Germania e l'Italia: la prima nel 2014 segnava un livello di convergenza di circa il $25 \%$ superiore a quello raggiunto nel 1980, la seconda del $30 \%$ inferiore.

La singolare traiettoria dell'Italia attesta un declino che è sia relativo, in comparazione con i suoi pari, sia assoluto, in comparazione con le sue passate prestazioni. Naturalmente è solo durante la Grande Recessione che il paese ha conosciuto un declino prolungato del livello della PTF, che sino alla crisi del 2008 continuò comunque a crescere - 
seppure, come si è detto, a una velocità inferiore a quella alla quale avanzava la frontiera. Ma ciò che conta, specialmente in un contesto internazionale marcato da una sempre più intensa globalizzazione, è proprio la capacità di un'economia di rimanere prossima alla frontiera. L'Italia pare averla perduta. Vecchi (2017, Fig. 7.8) compara l'andamento della crescita pro capite in Italia nel periodo 1861-2013 con quello degli altri stati membri dell'OCSE, che riunisce le tre dozzine di economie più avanzate del pianeta. Egli conclude che il declino dell'Italia è solo relativo, «per ora» (p. 289). Ma se la tendenza della PTF non s'invertirà non passerà molto tempo prima che esso si volga in declino assoluto.

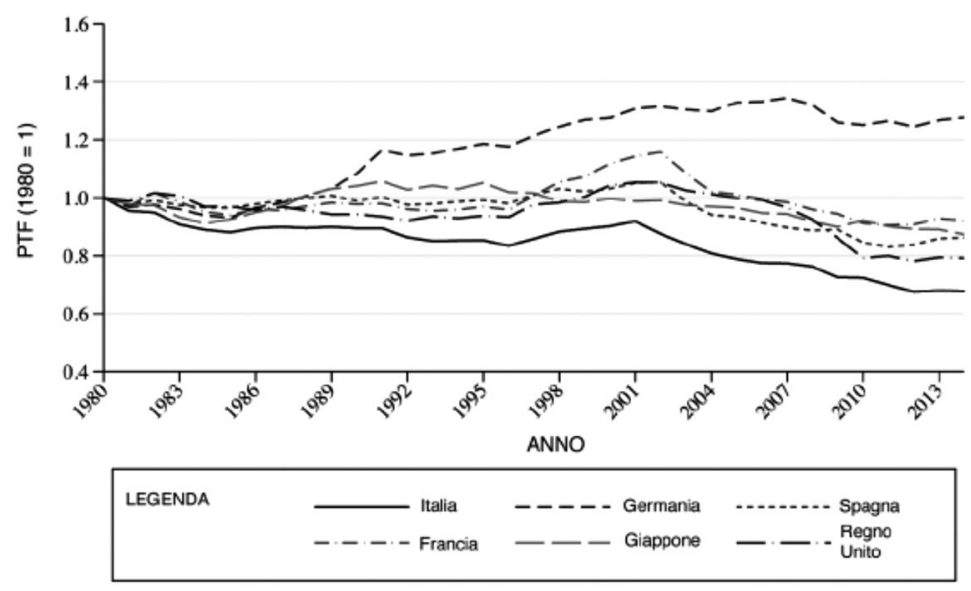

Fonte: Penn World Table

Fig. 2 - Andamento comparato della PTF, 1980-2014.

Fu infatti soprattutto la dinamica della PTF a spingere la lunga rincorsa dell'Italia verso la prosperità. Lo dimostra la Fig. 3, che ripartisce i contributi forniti alla crescita economica dall'aumento della popolazione, dall'accumulazione di capitale, e dall'avanzamento della PTF (la Figura deriva da un esercizio standard di contabilità della crescita: un'analisi più sofisticata è in Broadberry et al. 2013). Essa copre i sette decenni durante i quali l'Italia divenne un'economia industriale avanzata, e li ripartisce secondo due periodizzazioni alternative: quella ortodossa (Fig. 3b), e quella che spezza in due l'Età dell'Oro ed enfatizza il picco che l'Italia toccò nel 1980. 
A

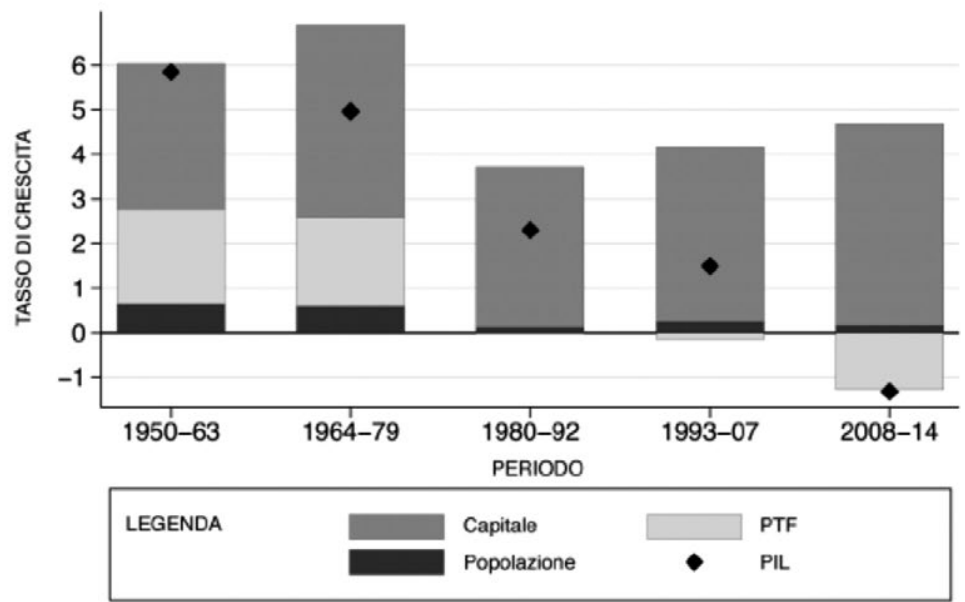

Fonte: Penn World Table

B

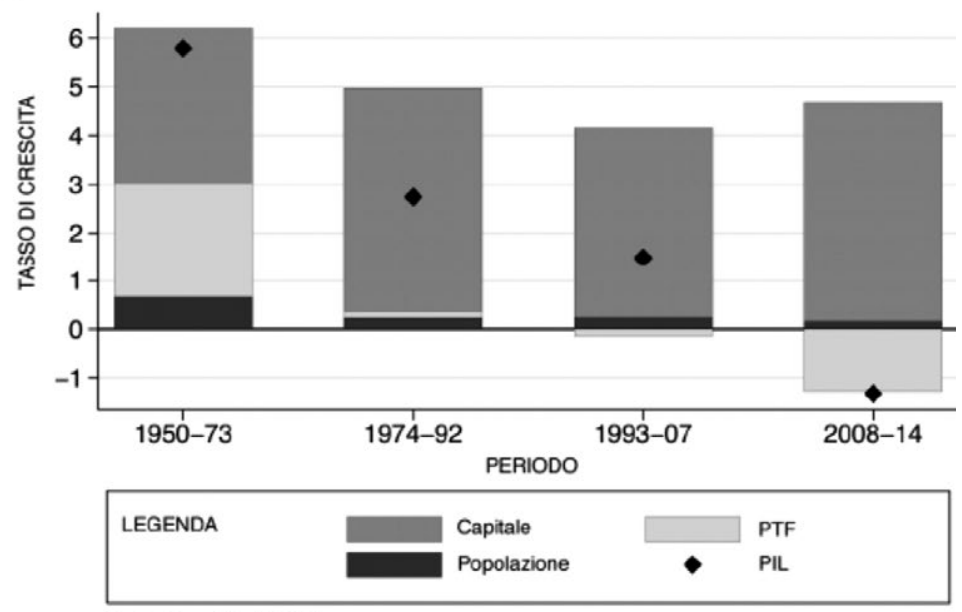

Fonte: Penn World Table

Fig. 3 - Contributo della PTF alla crescita, 1950-2014.

Il contributo della PTF all'espansione dell'economia italiana fu elevato e crescente nel periodo durante il quale le potenzialità del 
modello di crescita fondato sul catch-up - cioè il modello, tipico di economie relativamente arretrate, nel quale la crescita economica è alimentata soprattutto dal trasferimento di risorse dall'agricoltura all'industria e ai servizi ad essa collegati, e dalla parallela adozione di superiori tecnologie straniere - erano maggiori, ossia fino al raggiungimento della frontiera della produttività, e poi decrebbe. Mentre l'Italia convergeva sulla frontiera e parallelamente diminuiva il potenziale della crescita di catch-up, il contributo dell'innovazione tecnologica e organizzativa endogena avrebbe dovuto progressivamente aumentare; il fatto che nel corso degli scorsi tre o quattro decenni il contributo della PTF sia invece calato suggerisce che la transizione da quel modello di crescita a uno maggiormente fondato sull'innovazione di frontiera sia stato insufficiente. In altre parole, l'Italia seppe efficacemente sfruttare il vantaggio della propria relativa arretratezza, recuperando molto rapidamente il terreno che la separava dalle economie di più antica industrializzazione, ma non seppe completare la propria trasformazione in grado sufficiente da agganciarsi stabilmente alla frontiera della produttività.

Gli effetti furono compensati da tassi di accumulazione di capitale generalmente elevati (visibili nella Fig. 3a), sostenuti anche dalla crescita della spesa pubblica, e da una lenta ma costante crescita del tasso di partecipazione della popolazione adulta al mercato del lavoro. Mentre la sua capacità di combinare capitale e lavoro in modo sempre più efficiente deteriorava, dunque, l'Italia sostenne la propria crescita gettando sempre più investimenti e lavoratori nella fucina della produzione. $\mathrm{Ma}$ questa non è una strategia sostenibile nel lungo periodo, come si è detto, e già prima della Grande Recessione poté alleviare solo modestamente gli effetti della flessione della crescita della PTF: come indica la Fig. 3a, infatti, dopo la svolta degli anni ottanta la crescita aggregata rallenta sensibilmente nonostante che il ritmo di accumulazione del capitale acceleri. Né questo fenomeno può essere interamente attribuito alla più generale flessione dei ritmi di crescita del prodotto e della produttività in tutto il mondo occidentale, perché, come abbiamo visto, nel quindicennio precedente la crisi del 2008 la crescita pro capite fu pari a circa la metà del tasso medio dell'Eurozona.

L'esito è che, dopo il colpo inferto dalla Grande Recessione, il reddito reale medio disponibile è ora di qualche punto percentuale più basso di quanto non fosse nel 1995, come si è detto, mentre in Francia, Germania e Spagna esso è di circa venticinque punti più alto (Fmi 2017, 4). La recente epidemia virale - che nel momento in cui scrivo 
queste righe è ancora nella fase ascendente della curva - minaccia dunque di colpire duramente una società e un'economia italiana già fortemente indebolite.

\section{UN'INTERPRETAZIONE ISTITUZIONALE}

Come scrive Toniolo $(2013,40)$, l'Italia «sembra avere in qualche modo perso la sua "capacità sociale di crescita"». La questione è perché ciò sia avvenuto. La teoria istituzionale potrebbe offre una risposta plausibile.

L'investimento e l'innovazione sono le cause prossime della crescita, non le sue determinanti profonde. Come scrissero North e Thomas $(1973,2)$, esse «non sono cause della crescita: esse sono la crescita». Quindi cosa determina la crescita? cosa spinge alcuni paesi a investire e innovare più di altri? Questa domanda ha ricevuto quattro principali risposte: la geografia, e quindi il clima di un paese, la sua vulnerabilità a malattie endemiche, la sua dotazione di risorse naturali, la sua prossimità alle principali vie di comunicazione (e.g., Diamond 1997); l'integrazione nei mercati internazionali (e.g., Frankel e Romer 1999); la cultura, la religione, e i convincimenti diffusi, sulla traccia della tesi di Max Weber (e.g., Landes 1998); e le 'istituzioni'.

La definizione più comunemente accettata della nozione di 'istituzioni' le descrive come le «regole del gioco» di una società, ossia il quadro all'interno del quale si svolgono gli scambi economici, sociali e politici (North 1994, 360):

le istituzioni sono quei vincoli, creati dall'uomo, che strutturano le interazioni umane. Esse consistono di vincoli formali (ad esempio regole, leggi, costituzioni), di vincoli infornali (ad esempio norme di comportamento, convenzioni sociali, codici di condotta), e del modo in cui entrambi sono applicati [their enforcement characteristics]. Insieme essi definiscono la struttura degli incentivi di una società, e più specificamente di un'economia.

Ed è appunto in virtù della loro capacità di incidere sugli incentivi a investire e innovare che una vasta e crescente letteratura (e.g., North 1990, 1994; Acemoglu et al. 2001) afferma che, nel lungo periodo, la qualità delle istituzioni di un paese è una determinante decisiva della crescita, sulla quale esercita un'influenza maggiore che la geografia, l'in- 
tegrazione nel commercio internazionale, o le tradizioni culturali.

Un'utile illustrazione di questa teoria sta nel saggio che Baumol (1990) dedica al tema, cruciale, dell'allocazione tra diversi usi possibili del 'talento imprenditoriale' presente in una popolazione. La sua tesi (p. 918) è che le istituzioni «che determinano il rendimento delle diverse attività imprenditoriali svolgono un ruolo decisivo nell'allocare $i$ talenti imprenditoriali tra usi produttivi e usi improduttivi». Quindi, qualora una società sia retta da istituzioni che riservano al procacciamento di rendite e privilegi, all'evasione fiscale, o al crimine organizzato - sono gli esempi usati da Baumol - un rendimento superiore a quello che si può ottenere dall'impegno nell'innovazione industriale, i potenziali imprenditori presenti nella popolazione si dirigeranno verso quelle attività improduttive in proporzioni maggiori che in società nelle quali il rapporto tra i due rendimenti sia l'inverso. E se quella società riformasse le proprie istituzioni in modo tale da ridurre i rendimenti delle attività socialmente indesiderabili, essa, a parità di ogni altra condizione, beneficerebbe di un aumento del flusso di talento imprenditoriale verso gli 'usi produttivi' e, con esso, di un'accelerazione del ritmo di crescita di lungo periodo.

\section{DUE OBIEZIONI}

Per quanto plausibile, se applicata al caso italiano questa lettura si presta a due evidenti obiezioni. La prima è che, a partire dai primissimi anni Novanta l'Italia ha avviato un vasto programma di riforme, che ha toccato molte delle più importanti «regole del gioco», sia nel settore privato - si pensi alle riforme dei mercati finanziari, per esempio, o del governo delle imprese - sia nel settore pubblico, e la cui ispirazione generale fu largamente condivisa: perché la crescita della PTF avrebbe rallentato proprio mentre la società migliorava (quantomeno nel complesso) la qualità delle proprie istituzioni? La seconda obiezione, simile, si rivolge al passato. La sua premessa è che, qualunque sia il giudizio sulle riforme del passato quarto di secolo, è indubbio che le istituzioni del paese fossero significativamente peggiori nel periodo - il ventennio successivo all'ultima guerra - durante quale le prestazioni dell'economia furono più brillanti: le curve della PTF e della qualità delle istituzioni suggerirebbero dunque una relazione inversa a quella postulata dalla letteratura, nella quale a istituzioni deteriori corrisponde 
crescita elevata, e al migliorare della qualità delle istituzioni si accompagna un rallentamento della crescita.

Per rispondere alla prima obiezione conviene tornare sulla nozione stessa di 'istituzioni', citata sopra, che è composita: North (p. 366) la completa chiarendo che «è la mescolanza [admixture] tra le regole formali, le norme informali, e i modi in cui esse sono applicate che determina le prestazioni di un'economia». Ciò che conta, in altre parole, è il modo in cui le istituzioni effettivamente incidono sugli incentivi di imprese, cittadini, autorità pubbliche: dove le leggi scritte sono spesso trascurate a favore delle norme sociali, sulle scelte di questi attori incideranno più le seconde che le prime. Sicchè un ampio scarto può esistere tra le istituzioni formali di una società, quelle scritte nelle leggi, e le loro istituzioni reali, quelle che effettivamente guidano le scelte dei cittadini e delle imprese. Del resto, i tre esempi di attività socialmente indesiderabili che Baumol (1990) usa - il procacciamento di rendite e privilegi, l'evasione fiscale, il crimine organizzato - sono altrettanti esempi di questo scarto: l'evasione fiscale, in particolare, è una violazione delle leggi scritte, che può diffondersi largamente - e, nell'esempio di Baumol, offrire elevati rendimenti - solo se le norme sociali la tollerano e l'applicazione delle leggi scritte è inadeguata.

La risposta alla prima obiezione è dunque che le riforme del passato quarto di secolo hanno cambiato le istituzioni reali dell'Italia in grado minore di quanto ne abbiano cambiato le leggi scritte: come suggerirà la Fig. 4, sul grado nel quale le leggi sono generalmente rispettate, quelle riforme sono riuscite solo in parte a modificare gli incentivi che concorrono a determinare le scelte di imprese, famiglie, e autorità pubbliche.

Sul piano teorico, la risposta alla seconda obiezione si può trarre altrettanto agevolmente dalla versione Schumpeteriana della teoria della crescita, che si è progressivamente aperta ai risultati raggiunti dagli studi sulle istituzioni, formulando una teoria delle istituzioni 'appropriate' (Acemoglu et al. 2006; Aghion et al. 2014).

Il 'vantaggio dell'arretratezza' di cui scrisse Gerschenkron (1962) deriva dall'ovvia constatazione che importare e adattare tecnologie già sperimentate è più semplice che sviluppare nuove tecnologie superiori a quelle esistenti. Di conseguenza economie comparativamente arretrate, quale era l'Italia del secondo dopoguerra, possono crescere più rapidamente di economie che abbiano già raggiunto la frontiera della produttività perché possono assorbire le tecnologie sviluppate da queste ultime: ciò consente loro di ottenere aumenti di produttività che, in un 
medesimo periodo di tempo, sono tipicamente superiori a quelli derivanti dall'innovazione di frontiera. Il ritmo di crescita della produttività espresso da un'economia relativamente arretrata può quindi essere stabilmente superiore a quello di economie più avanzate, alimentando un processo di convergenza - catch-up, lo abbiamo anche definito - che gradualmente rallenta, man mano che la frontiera si avvicina, e si conclude quando questa è raggiunta: allora la dinamica della produttività tende ad allinearsi a quella delle economie più avanzate, la quale dipende dal più arduo sforzo di spingere in avanti la frontiera tecnologica. Tuttavia Gerschenkron osservò che le politiche economiche e le istituzioni che favoriscono l'importazione, l'adattamento e la diffusione di tecnologie straniere già sperimentate non sono necessariamente le più adeguate a stimolare anche l'innovazione di frontiera, e vice versa. Quindi è possibile che a diversi fasi dello sviluppo di un'economia ossia a diversi stadi del processo di convergenza - debbano corrispondere istituzioni diverse.

Acemoglu et al. (2006) hanno dato conferma empirica di questa ipotesi verificandola sopra un vasto campione di paesi arretrati e avanzati. Essi impiegano due variabili istituzionali, scelte per l'influenza che ciascuna esercita sull'intensità della concorrenza: una misura dell'apertura di un'economia al commercio internazionale, e una misura dell'apertura dei mercati interni all'accesso di nuove imprese. Le loro conclusioni suggeriscono che nelle economie prossime alla frontiera la concorrenza svolge un ruolo particolarmente importante nello spingere la crescita, mentre in economie comparativamente arretrate la relazione tra crescita e concorrenza appare più debole e ambigua (Aghion et al. 2014). Simili ricerche sono state compiute sulla rule of law, la supremazia della legge, giungendo a conclusioni simili: l'effetto positivo che questa (macro)istituzione esercita sulla crescita economica - favorendo la crescita dimensionale delle imprese, la distruzione creatrice, la riallocazione di capitale e lavoro da imprese meno produttive a imprese più produttive - appare più forte in economie prossime alla frontiera che in economie relativamente arretrate.

Dunque mentre un'economia progredisce nel proprio processo di convergenza verso la frontiera della produttività ci si attenderebbe di osservare una parallela e tendenzialmente contemporanea evoluzione nelle sue istituzioni, e in particolare nelle due - protezione della concorrenza e supremazia della legge - appena menzionate, così da agevolare la transizione da un modello di crescita di catch-up, fondato sul 
cambiamento strutturale e l'importazione di tecnologia, a uno maggiormente dipendente dall'innovazione endogena e di frontiera, che necessariamente implica tassi maggiori di distruzione creatrice, grazie alla quale le nuove innovazioni rimpiazzano quelle passate e dissipano le rendite che esse hanno creato.

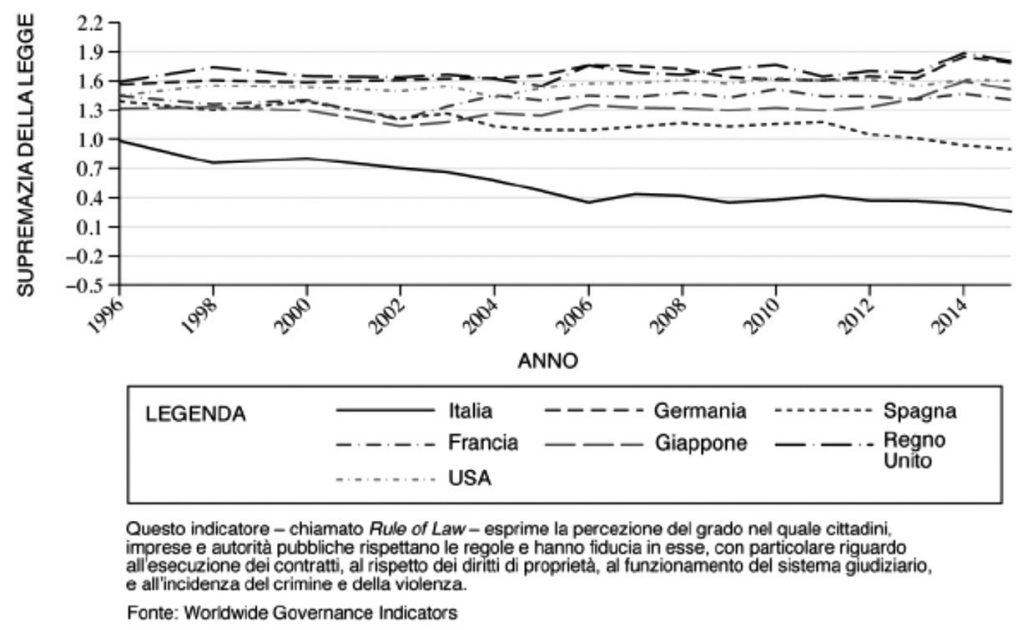

Fig. 4 - Indicatore della supremazia della legge (rule of law), 1996-2015.

Come indica la Fig. 4, tuttavia, in Italia la supremazia della legge appare debole; e l'adozione di una moderna politica della concorrenza fu tardiva ed ebbe effetti modesti (Giordano e Zollino 2017). Quindi sotto due aspetti cruciali le istituzioni del paese potrebbero essere inadeguate al suo stadio di sviluppo: nel lessico di Acemoglu et al. (2006) e Aghion et al. (2014), l'Italia avrebbe istituzioni 'inappropriate'. Secondo la teoria che abbiamo appena riassunto, esse non avrebbero significativamente frenato la crescita durante i primi due o tre decenni dal secondo dopoguerra, e potrebbero anzi averla favorita, ma progredendo il progresso della convergenza del paese, mentre l'impeto del cambiamento strutturale si affievoliva e il vantaggio dell'arretratezza scemava, tali difetti istituzionali divennero vincoli di crescita sempre più stringenti, che ritardarono e ostacolarono l'attivazione di motori di crescita più adeguati a un'economia avanzata. Sicché la risposta alla seconda obiezione è che in Italia le istituzioni sono forse migliorate ma 
non abbastanza, sia perché il paese aveva bisogno di avvicinarsi a un nuovo modello di crescita, più adatto a un'economia ormai prossima alla frontiera della produttività, sia perché in parallelo cambiavano sia il contesto tecnologico (la rivoluzione delle ICT) sia le sfide e le opportunità che l'economia internazionale presentava (con la seconda globalizzazione, la crescita della Cina e di altre economie emergenti, l'unione monetaria europea).

IL PROBLEMA DELL'AZIONE COLLETTIVA: GIOCHI DI COORDINAMENTO E DILEMMI DEL PRIGIONIERO

Abbiamo sinora inteso la supremazia della legge - la rule of law come la tendenziale coincidenza tra le istituzioni formali di una società e quelle reali. In modo più vigoroso, ma del tutto equivalente, Hoff e Stiglitz $(2008,1474)$ la definiscono come ciò che «impedisce ai pochi di rubare ai molti». La supremazia della legge è infatti una protezione il cui valore è tanto più elevato quanto più debole è il potere - politico, economico, sociale - del singolo; ed è altrettanto evidente che la supremazia della legge non può restare debole, nel lungo periodo, se le autorità pubbliche sono soggette a forte responsabilità politica, tale da allineare i loro incentivi agli interessi della maggioranza dei cittadini.

Conviene allora chiedersi quale logica spieghi la persistenza di un fenomeno che, per la maggior parte della società, è indesiderabile. Per farlo useremo un esempio semplificato delle interazioni tra cittadini, politici e stato che ruotano attorno alla fornitura dei beni e dei servizi che la società si attende di ricevere dalle autorità pubbliche. Lo scopo è mostrare come il debole rispetto della legge e la debole responsabilità politica delle autorità pubbliche possano rafforzarsi reciprocamente e cristallizzarsi in un equilibrio stabile e persistente.

In una democrazia contemporanea queste interazioni coinvolgono due scambi. Uno è lo scambio tra la fornitura dei beni e dei servizi pubblici e il pagamento delle imposte. Immaginiamo, a questo proposito, che i beni e i servizi forniti dalle autorità pubbliche possano essere distribuiti a tutti i cittadini secondo criteri equi e imparziali, come la legge generalmente richiede (e definiremo 'servizi pubblici' i beni e i servizi così distribuiti), ovvero possano essere distribuiti ad alcuni cittadini solamente, secondo criteri mirati e particolaristici (li definiremo 'servizi privati', per quanto anch'essi derivino dalle risorse pubbliche). 
Il vincolo di bilancio limita l'ammontare totale dei servizi, 'pubblici' o 'privati', che ogni anno lo stato può distribuire alla società. Anche i cittadini operano sotto un vincolo di bilancio. Essi possono evadere le imposte, per migliorare il proprio standard di vita destinando i soldi non versati allo stato a consumi o investimenti che altrimenti non sarebbero stati possibili, ma così facendo si espongono al rischio di sanzioni che, tipicamente, sono maggiori del guadagno.

L'altro scambio è quello tra il valore dei servizi pubblici forniti ai cittadini - valore valutato, come vedremo, in relazione alle imposte da essi pagate - e le loro scelte politiche. Le preferenze dei cittadini quanto alla quantità e alla natura dei servizi pubblici che lo stato dovrebbe fornire alla società sono le più varie, naturalmente, così come diverse sono le opinioni sul livello e sulla forma di imposizione fiscale più adeguati a finanziare i servizi pubblici. Ci concentreremo invece sulla sola questione dell'equivalenza tra servizi pubblici ricevuti e imposte versate, rispetto alla quale possiamo presumere che le preferenze dei cittadini siano uniformi: a parità di ogni altra condizione, essi sosterranno i politici che siano ritenuti capaci di assicurare quell'equivalenza, e avverseranno i politici che non lo sono.

Esaminiamo un caso nel quale l'equivalenza si rompa. Inizialmente l'intero bilancio dello stato è distribuito sotto forma di servizi pubblici, come li abbiamo definiti; tutti i cittadini pagano le imposte dovute; e i due flussi sono giudicati equivalenti. Ma una causa esogena crea un'inefficienza nella distribuzione dei servizi pubblici, che assorbe parte del bilancio pubblico e pertanto provoca una riduzione dell'ammontare totale dei servizi pubblici distribuiti ai cittadini, con la conseguenza che ciascuno di essi riceverà un paniere di servizi pubblici il cui valore è inferiore alle imposte versate allo stato.

I cittadini hanno due strade per reagire alla rottura dell'equivalenza. Possono continuare a pagare le imposte dovute, e in parallelo esercitare pressione sul sistema politico - tramite azioni quali informarsi, votare, scrivere petizioni, manifestare: azioni che per ora immaginiamo siano giudicate costose, per il dispendio di tempo ed energia che comportano - per domandare che l'equivalenza sia ristabilita (assumiamo che politiche innovative possano ristabilirla nel medio periodo, ma che nel breve termine l'individuazione delle politiche adeguate sia incerta). Ovvero possono ricorrere a rimedi di natura privata: evadere le imposte, quantomeno in misura sufficiente ad acquistare sul mercato la porzione di servizi pubblici che lo stato ha smesso di fornire; corrompere 
politici o funzionari pubblici, per ricevere servizi privati ovvero per occultare l'evasione fiscale; o combinare assieme queste due strategie. A loro volta, i politici hanno due opzioni. Dovranno scegliere se accettare il prezzo della corruzione e il sostegno politico clientelare che l'accompagna, ovvero rifiutarli e riporre le proprie speranze di successo sulla (incerta) ricerca di politiche innovative che siano in grado di ristabilire l'equivalenza perduta. Naturalmente, anche la corruzione comporta un rischio di sanzioni per entrambi i protagonisti.

Rispetto a queste scelte, assumiamo che esistano due tipi di cittadini e di politici. Esistono politici che sempre rifiutano la corruzione, il clientelismo, e la fornitura particolarista delle risorse pubbliche (che chiameremo politici 'virtuosi', imponendo però a questo termine un senso puramente descrittivo e non normativo). Ed esistono politici che invece decidono di volta in volta se accettare tangenti, tollerare l'evasione fiscale o fornire servizi privati, a seconda che i benefici di queste scelte eccedano o meno i costi (li chiameremo politici 'opportunistici', nel medesimo senso). Specularmente, esistono cittadini che sempre preferiscono pagare le imposte dovute ed esercitare attivamente i loro diritti politici (cittadini 'virtuosi'), e cittadini che decidono di volta in volta comparando $i$ costi e i benefici di questa opzione con i costi e $i$ benefici dell'opzione fondata sui rimedi privati (cittadini 'opportunistici'). Assumiamo che anche gli opportunisti, cittadini e politici, attribuiscano un valore alle virtù civiche; ma a differenza dei cittadini e dei politici virtuosi essi sono pronti a trascurarle se i benefici della scelta opportunistica eccedono i costi in misura maggiore di un dato margine, variabile da persona a persona: un margine che possiamo intendere come la traduzione monetaria del costo morale della violazione dei propri convincimenti normativi. Possiamo quindi immaginare un continuum, tra opportunisti che devieranno dai propri convincimenti normativi solo se il beneficio netto che ne deriva è molto alto, la cui posizione è molto vicina a quella delle persone virtuose, e opportunisti pronti a ignorare la legge morale che è in loro anche per vantaggi materiali molto esigui. Tutti i membri della società sanno che esistono cittadini e politici di entrambi i tipi, ma tutti ignorano chi siano le persone virtuose e chi siano gli opportunisti, e altresì ignorano, tra questi ultimi, quale sia la soglia oltre la quale ciascuno è pronto a violare i propri convincimenti normativi. A parità di ogni altra condizione, pertanto, sino a che le loro azioni non sono scoperte i politici che agiscono opportunisticamente hanno le medesime possibilità di raccogliere consenso che i 
politici (virtuosi od opportunistici) che agiscono virtuosamente: quindi accettando la corruzione e il sostegno clientelare che l'accompagna i politici che agiscono opportunisticamente acquisiscono un vantaggio competitivo sui politici che agiscono virtuosamente.

La risposta della società alla rottura dell'equivalenza tra imposte versate e servizi pubblici distribuiti dipende dall'entità dello scarto tra i due flussi. Se la diminuzione dei servizi pubblici distribuiti ai cittadini è sufficientemente ampia da indurre almeno un cittadino opportunista e un politico opportunista a violare i propri doveri pubblici (ossia, è sufficientemente ampia da spingere i benefici dell'opportunismo oltre la loro soglia dell'immoralità), allora qualche soldo dovuto allo stato non sarà versato, qualche servizio privato sarà fornito, e sorgerà una relazione clientelare. Ciò ridurrà il bilancio pubblico, e ridurrà pertanto i servizi pubblici distribuibili l'anno successivo, allargando ulteriormente il divario tra le imposte pagate dai cittadini e i servizi pubblici da essi percepiti. E siccome il beneficio netto della violazione delle regole cresce al crescere del divario, esso supererà la soglia di altri cittadini e politici, che sceglieranno la risposta opportunistica. Quindi l'anno successivo lo scarto crescerà ulteriormente, col medesimo effetto. $\mathrm{E}$ così via. Una volta innescata, anzi, questa dinamica accelererà. Sia perché al crescere del numero degli opportunisti che scelgono quella via decresce il rischio di essere scoperti, e quindi cresce il beneficio netto di quella scelta. Sia perché la progressiva riduzione dei servizi pubblici distribuiti segnalerà alla società che un numero crescente di opportunisti ha scelto quella via, e ciò indurrà altri opportunisti ad anticipare la propria reazione: ossia ad agire opportunisticamente prima del raggiungimento della propria soglia, in previsione che presto sarà superata; perché per un opportunista sarebbe irrazionale comportarsi virtuosamente quando avesse ragione di prevedere che, a causa della marea montante dell'opportunismo, l'anno successivo molto probabilmente riceverà un ammontare di servizi pubblici di valore inferiore alla somma tra le imposte versate e la propria soglia dell'immoralità.

Se dunque gli opportunisti perseguono razionalmente il proprio interesse individuale, al crearsi di uno scarto sufficientemente ampio tra imposte pagate e servizi pubblici distribuiti seguirà una spirale ad esito della quale la società si assesterà su un nuovo equilibrio, nel quale solo i cittadini virtuosi pagheranno le imposte, solo i politici virtuosi rifiuteranno corruzione e clientelismo, e i politici opportunistici prevarranno largamente sui virtuosi (perché meno esposti alla responsabilità politica 
grazie alla disponibilità di maggiori fondi, frutto della corruzione, e di una solida base clientelare di consenso). A parte le conseguenze sulla diseguaglianza economica, che possono essere significative ma qui tralasceremo, spostandosi su questo equilibrio la società si sarà impoverita (a parità di ogni altra condizione): perché la diffusione della corruzione e dell'evasione fiscale tipicamente riduce l'efficienza dell'allocazione delle risorse, perché la capacità dello stato di fornire servizi pubblici essenziali (la sicurezza interna ed esterna, per esempio) potrebbe essere compromessa, e perché al prevalere dei politici opportunisti - il cui successo dipende solo in parte dalla bontà delle politiche proposte e dalla coerenza dei loro comportamenti - la qualità delle politiche pubbliche tenderà a deteriorarsi.

Tipicamente questo equilibrio sarà persistente, perché nel corso del tempo la diffusione di comportamenti opportunistici tenderà a modificare non solo i convincimenti fattuali dei cittadini - le loro aspettative quanto a come i loro pari e la società intera risponderanno alle scelte (virtuose o opportunistiche) di ciascuno - ma anche i loro convincimenti normativi, che gradualmente si adegueranno alla realtà osservata. Infatti, al crescere della diffusione di comportamenti opportunistici tenderanno a decrescere il civismo, la fiducia generalizzata, la legittimità delle leggi, l'indignazione a vederle violate, e lo spontaneo contributo dei cittadini alla loro osservanza e sorveglianza. Segnalando che la società ha abbandonato le regole e i valori sui quali i cittadini avevano ritagliato i propri convincimenti normativi, anch'essi cambieranno, almeno presso alcune persone: e l'effetto del cambiamento non sarà altro che di abbassare la soglia degli opportunisti o di trasformare in opportunisti le persone virtuose (il che permette di superare la comoda ma artificiale bipartizione tra virtuosi e opportunisti). E naturalmente il cambiamento dei convincimenti normativi innescherà nuove fasi della spirale discendente, che spingeranno l'equilibrio sempre più in basso.

Se questo è vero sorgono due domande. Se la conseguenza è la spirale distruttiva che abbiamo appena descritto, davvero il comportamento opportunistico fu una reazione razionale all'emergere di uno scarto tra imposte e servizi pubblici? E a quali condizioni la società può evitare questa spirale, come le contemporanee società occidentali generalmente riescono a fare? Iniziamo dalla prima domanda, che è rilevante anche dal punto di vista degli interessi individuali dell'opportunista: perché il più felice di essi è quello che è il solo a comportarsi opportunisticamente, e viceversa. È sicuramente giusto «mantenere i 
patti», dice il «pazzo» di Hobbes (Leviatano, XV.4), ma se tutti mantengono i patti e così pongono termine alla guerra di tutti contro tutti, perché non sarebbe razionale per me violarli, se ne posso trarre un beneficio? Allo stesso modo, l'unico evasore fiscale della società potrà godere di una fornitura di servizi pubblici pressoché piena, perché tutti gli altri cittadini continuano a pagare le imposte, e potrà dedicare tutti i soldi così risparmiati - dedotto il prezzo della corruzione, per proteggersi dal rischio di essere punito - a elevare la qualità materiale della sua vita.

Il nocciolo di questo esempio è un problema di azione collettiva. Il dilemma sta nel fatto che l'equilibrio iniziale, quello precedente la rottura dell'equivalenza tra imposte e servizi pubblici, è preferibile per tutti, sicché in un referendum tutti i membri della società voterebbero in suo favore (Sen 1967); ma il comportamento opportunistico, che può innescare la discesa all'equilibrio deteriore, può nondimeno essere conforme all'interesse individuale. La questione cruciale è comprendere se la strategia opportunistica sia sempre nell'interesse individuale, qualunque sia la reazione del resto della società (ossia a prescindere da quale sia l'equilibrio, buono o deteriore, sul quale la società si assesterà in conseguenza della scelta opportunistica della persona in questione). Per esaminare questo tema conviene definire la nozione di interesse individuale: lo intenderemo come l'interesse oggettivo e di lungo periodo di una persona, tenendo conto pertanto anche della probabilità e dei verosimili effetti della discesa all'equilibrio deteriore.

Se il comportamento opportunistico è sempre nell'interesse individuale, che è la caratteristica del gioco noto come il 'dilemma del prigioniero', la teoria della scelta razionale basata sul calcolo dei costi e dei benefici predice che la spirale generalmente si innescherà. In questo contesto, comportamenti virtuosi possono derivare solo da coercizione, tramite l'imposizione di leggi adeguate e coerentemente attuate (leggi motivate dall'interesse della società di preservare, o raggiungere, il buon equilibrio). Come Basu (2015) osserva, tuttavia, la coercizione sarà efficace solo se i pubblici ufficiali si comporteranno virtuosamente. Ma siccome non è possibile presupporre un comportamento virtuoso da parte loro, perché anch'essi sono parte della medesima società, esposti ai medesimi incentivi degli altri cittadini, e potrebbero anch'essi scegliere di comportarsi opportunisticamente, la soluzione della coercizione è dubbia. Nel nostro esempio, inoltre, i politici opportunistici gradualmente prevalgono sui politici virtuosi, ed è inverosimile che essi 
favoriranno la rigorosa attuazione di quelle medesime leggi sulla cui violazione il loro successo politico ed economico è fondato.

La logica cambia se il comportamento opportunistico non è sempre nell'interesse individuale. Ad esempio, è possibile che il comportamento virtuoso sia conforme all'interesse individuale a condizione che tutta la società -0 , più realisticamente, la vasta maggioranza dei cittadini - si comporti in modo virtuoso. È questo il caso delle risorse comuni, per esempio, che possono essere distrutte dallo sfruttamento eccessivo. In questa situazione, le scelte di ciascuno dipenderanno dalle sue aspettative rispetto al comportamento del resto della società. Questa è la situazione descritta dal gioco di assicurazione, o di coordinamento, che ha due equilibri. Ossia, se gli opportunisti si aspettano che il resto della società si comporterà virtuosamente, si comporteranno virtuosamente anch'essi e la società resterà sul buon equilibrio, che è preferibile per tutti; nel caso opposto essi si comporteranno opportunisticamente, e la società scivolerà sull'equilibrio deteriore. In tal caso per evitare la spirale discendente la coercizione non è necessaria, a rigore: è sufficiente coordinare le aspettative dei cittadini. Come Ostrom (2000) rilevò, norme sociali fondate sulla cooperazione e la reciprocità sono spesso in grado di risolvere problemi di azione collettiva di questo genere. La norma sociale impiegata per coordinare le aspettative della società nella direzione del buon equilibrio deve naturalmente essere credibile ex ante, per guidare le scelte degli opportunisti, ma deve anche essere poi confermata dalla realtà, dagli esiti effettivamente sperimentati: perché se, contrariamente alle aspettative ingenerate dalla norma sociale, la gran parte della società poi non si comportasse virtuosamente, gli opportunisti che avessero scelto di pagare le imposte riceverebbero una quantità di servizi pubblici inferiore a quella attesa al momento del calcolo dei costi e dei benefici: subirebbero quindi una perdita, che non avrebbero subito se si fossero comportati opportunisticamente. Rilevata l'inaffidabilità delle aspettative generate dalla norma sociale di cooperazione, è presumibile che all'occasione successiva gli opportunisti che ne hanno subito una perdita si comporteranno opportunisticamente. Ciò innesca la spirale. Gli opportunisti del nostro esempio ne sono consapevoli, naturalmente, perché sono in grado di proiettare sugli altri il medesimo ragionamento che li ha condotti a comportarsi opportunisticamente; e sono altrettanto consapevoli che l'equilibrio buono è desiderabile anche per loro. Essi si comportano opportunisticamente per limitare le perdite, letteralmente, non per lucrare un vantaggio. 
È in questo senso che per il cittadino comune può essere razionale accettare la corruzione spicciola o piegarsi al clientelismo, nonostante il danno che questi fenomeni provocano alla società. Queste sono strategie difensive, adottate in reazione al prevalere - osservato, previsto, o temuto - dell'opportunismo. In altre parole, la logica della spirale apre una tensione tra le preferenze apparenti dei cittadini, quelle che sono rivelate dalle loro scelte opportunistiche, e le loro preferenze latenti, che invece aspirano al buon equilibrio e ai reciproci comportamenti virtuosi che lo rendono possibile.

Dalla logica del gioco di assicurazione, o di coordinamento, derivano due conseguenze. La prima è che se il comportamento virtuoso è conforme all'interesse individuale solo a condizione che la vasta maggioranza della società si comporti virtuosamente, allora la spirale discendente non s'innescherà se non quando la diffusione dei comportamenti opportunistici abbia raggiunto una soglia minima (che è il complemento all'intero della porzione di società che abbiamo definito 'vasta maggioranza'). La seconda è che il ritorno (o l'ascesa) al buon equilibrio è sempre possibile, se le aspettative diffuse nella società lo favoriscono, perché è sempre razionale rispondere con comportamenti virtuosi alla prevalenza nella società di comportamenti virtuosi. Ma anche la spirale ascendente dipenderà dal raggiungimento di una soglia minima, al di sotto della quale il circolo virtuoso non s'innesca: una soglia che non è necessariamente uguale a quella che mette in moto la spirale discendente.

Chiarita la differenza tra la logica del dilemma del prigioniero (operante quando il comportamento opportunistico è sempre nell'interesse individuale) e la logica del gioco di coordinamento (operante quando il comportamento opportunistico non è sempre nell'interesse individuale), occorre chiedersi quale delle due guidi le scelte della società. La questione dipende dalla valutazione degli interessi dei cittadini, così come li abbiamo definiti.

Una congettura plausibile è che entrambe le logiche siano operanti nelle società occidentali contemporanee, le quali del resto impiegano sia la coercizione - tipica della prima logica - sia il coordinamento tipico della seconda - per evitare la discesa all'equilibrio deteriore. La ragione sta nel diverso grado di potere politico ed economico di diversi strati della popolazione. Per almeno una parte delle élite, infatti, il comportamento opportunistico può essere una scelta razionale anche qualora esso inneschi la spirale discendente e ciò provochi un significativo 
danno alla società. Se la supremazia della legge è molto debole, per esempio, così come è probabile che sia nell'equilibrio deteriore, la grande evasione fiscale o la grande corruzione - quella che incide su appalti pubblici di elevato valore, o sulla regolazione di interi mercati o settori - possono assicurare a chi le pratica benefici di misura tale da eccedere il danno che essi subiscono a causa della discesa all'equilibrio deteriore. Ma è molto improbabile che ciò sia vero anche per $\mathrm{i}$ cittadini comuni, $\mathrm{i}$ quali hanno relativamente poche imposte da evadere, e generalmente non possono praticare altro che la corruzione spicciola. Per loro il danno derivante dalla discesa all'equilibrio deteriore è tipicamente superiore - spesso molto superiore, presumibilmente - ai vantaggi che possono procurarsi violando la legge.

Si può quindi ritenere che gli interessi individuali della vasta maggioranza dei cittadini generalmente coincideranno con l'interesse generale, mentre gli interessi individuali di almeno parte delle élite generalmente divergeranno dall'interesse generale. Ciò non significa che i membri delle élite siano necessariamente degli opportunisti, ma solo che per almeno parte di essi i comportamenti opportunistici sono tipicamente razionali, perché grazie al potere del quale dispongono essi possono ricavarne benefici maggiori della loro quota del mancato guadagno di efficienza dovuto al mancato completamento della transizione verso l'ordine sociale aperto (o, nel nostro esempio, della loro quota del decremento di efficienza dovuto alla discesa all'equilibrio deteriore).

Un'ulteriore conseguenza di questa differenziazione è che le élite hanno interesse a minimizzare i comportamenti opportunistici tra i cittadini comuni. Perché meno diffuso è l'opportunismo, meno in basso scenderà l'equilibrio della società, e minore sarà la perdita di efficienza, che anche le élite subiscono: quindi minimizzare i comportamenti opportunistici tra i cittadini comuni aumenta i benefici netti dei comportamenti opportunistici delle élite. Esse pertanto favoriranno la creazione di una sorta di doppio regime, nel quale i comportamenti opportunistici delle élite godono di ampia impunità, che è requisito imprescindibile per trarne benefici, ma i comportamenti opportunistici dei cittadini comuni sono trattati con rigore, per evitare che si diffondano oltre la soglia che innesca la spirale discendente. Di nuovo, questo doppio regime è tipico dell'ordine sociale chiuso, che non garantisce eguaglianza di diritti ai cittadini comuni; ed esso può almeno in parte sopravvivere in una società in transizione che si sia data le forme di una democrazia, prendendo le forme di sistematiche ma non dichiarate 
deviazioni dall'universalismo delle nuove leggi. Affinché il doppio regime sia efficace, tuttavia, l'impunità delle élite non deve essere visibile: altrimenti i comportamenti opportunistici si diffonderebbero anche tra i cittadini comuni. Le azioni visibili dei suoi membri più eminenti sono infatti capaci di guidare le aspettative della società: se i cittadini comuni vedessero che i comportamenti opportunistici sono prevalenti tra le élite, essi potrebbero cessare di attendersi comportamenti virtuosi da parte dei propri pari, e, conseguentemente, potrebbero iniziare essi stessi a comportarsi opportunisticamente. Quindi un sipario deve nascondere il doppio regime agli occhi della società. In questo contesto l'ipocrisia è l'omaggio che la vecchia allocazione del potere, ereditata dall'ordine sociale chiuso, rende alle nuove istituzioni formali; ed ha la funzione di isolare la dinamica che prevale tra le élite da quella che anima la vita del resto della società. Ma più marcato è il doppio regime, più i suoi tratti traspariranno attraverso il sipario, con la conseguenza che porzioni crescenti della società gradualmente allineeranno i propri comportamenti a quelli osservati tra le élite.

Queste considerazioni suggeriscono dunque una possibile interpretazione del malessere del paese (interpretazione che ho tentato di articolare in Capussela 2018, 2019). I difetti istituzionali che deprimono la crescita derivano sono in larga parte riassumibili nell'ampio scarto tra le istituzioni formali dell'Italia, generalmente adeguate, e le sue istituzioni reali, sensibilmente meno eque ed efficienti: scarto che è illustrato nella Figura 4. Questo scarto, a sua volta, è in larga misura spiegabile, da un lato, con la compresenza della logica tipica del dilemma del prigioniero e della logica tipica del gioco di coordinamento, e, dall'altro lato, con l'operare del paradigma di razionalità che, in presenza di diffusi comportamenti opportunistici, spinge cittadini e imprese comuni ad adottare strategie difensive, le quali spingono la società verso l'equilibrio deteriore. Siccome però il gioco di coordinamento ha equilibri molteplici, la risalita verso equilibri più equi ed efficienti è sempre possibile: essa infatti risponde alle preferenze latenti di cittadini e imprese comuni.

Se questa ipotesi apparisse convincente, la via per la soluzione dei principali problemi politici ed economici del paese sarebbe di fare emergere le preferenze latenti di cittadini e imprese comuni e farle prevalere sulle preferenze rivelate dalle loro scelte effettive. Il ruolo della discussione pubblica sarebbe allora decisivo, sia per diffondere nella società la consapevolezza della logica discussa sopra, sia per spingerla a rivedere le proprie preferenze. In questo, a loro volta, sono decisivi i 
segnali che le elite trasmettono alla società: in loro assenza, la spinta per invertire la dinamica discussa sopra potrò derivare solamente da una diffusa presa di coscienza dei cittadini e delle imprese comuni.

\section{BIBLIOGRAFIA}

Acemoglu, Daron, Philippe Aghion, e Fabrizio Zilibotti. 2006. 'Distance to Frontier, Selection, and Economic Growth'. Journal of the European Economic Association 4 (1): pp. 37-74.

Acemoglu, Daron, Simon Johnson, e James Robinson. 2001. 'The Colonial Origins of Comparative Development: An Empirical Investigation'. American Economic Review 91 (5): pp. 1369-401.

Aghion, Philippe, Ufuk Akcigit, e Peter Howitt. 2014. 'What Do We Learn From Schumpeterian Growth Theory?'. In Handbook of Economic Growth, a cura di Philippe Aghion and Steven Durlauf, Vol. 2B, pp. 515-63. Amsterdam: Elsevier.

Banca d'Italia. 2016. 'Relazione annuale, anno 2015-CXXII esercizio', Roma, $31 \mathrm{mag}$ gio. URL: https://www.bancaditalia.it/pubblicazioni/relazione-annuale/2015/ index.html.

Basu, Kaushik. 2015. 'The Republic of Beliefs: A New Approach to "Law and Economics"'. World Bank Policy Research Working Paper 7259. URL: http://documents.worldbank.org/curated/en/2015/05/24454378/republicbeliefs-new-approach-'law-economics'.

Baumol, William. 1990. 'Entrepreneurship: Productive, Unproductive and Destructive'. Journal of Political Economy 98 (5): pp. 893-921.

Broadberry, Stephen, Claire Giordano, e Francesco Zollino. 2013. 'La produttività'. In L'Italia e l'economia mondiale dall'unità a oggi, a cura di Gianni Toniolo, pp. 257-311. Venezia: Marsilio.

Capussela, Andrea. 2019. Declino. Una storia italiana. Roma: Luiss University Press.

Capussela, Andrea. 2018. The Political Economy of Italy's Decline. Oxford: Oxford University Press.

Diamond, Jared. 1997. Guns, Germs and Steel: The Fate of Human Societies. New York: Norton.

Fondo Monetario Internazionale (FMI). 2015a. 'Italy - 2015 Article IV Consultation'. Country Report n. 15/166, luglio. URL: https://www.imf.org/external/pubs/ cat/longres.aspx?sk=43046.0.

Fondo Monetario Internazionale (FMI). 2015b. 'Italy - Selected issues'. Country Report n. 15/167, luglio. URL: https://www.imf.org/external/pubs/cat/longres.aspx?sk=43047.0.

Fondo Monetario Internazionale (FMI). 2017. 'Italy - 2017 Article IV Consultation'. Country Report n. 17/237, luglio. URL: http://www.imf.org/en/Publications/ CR/Issues/2017/07/27/Italy-2017-Article-IV-Consultation-Press-Release-StaffReport-and-Statement-by-the-Executive-45139. 
Frankel, Jeffrey, e David Romer. 1999. 'Does Trade Cause Growth?'. American Economic Review 89 (3): pp. 379-99.

Gerschenkron, Alexander. 1962. Economic Backwardness in Historical Perspective, a Book of Essays. Cambridge MA: Harvard University Press.

Giordano, Claire, e Francesco Zollino. 2017. 'Macroeconomic estimates of Italy's markups in the long run, 1861-2012'. Banca d'Italia Quaderni di storia economica, n. 39. URL: https://www.bancaditalia.it/pubblicazioni/quaderni-storia/20170039/index.html? com.dotmarketing.htmlpage.language $=1$.

Hoff, Karla, e Joseph Stiglitz. 2008. 'Exiting a lawless state'. Economic Journal 118 (531): pp. 1474-97.

Landes, David. 1998. The Wealth and Poverty of Nations: Why Some Are So Rich and Some So Poor. New York: Norton.

North, Douglass. 1994. 'Economic Performance through Time'. American Economic Review 84 (3): pp. 359-68.

North, Douglass. 1990. Institutions, Institutional Change, and Economic Performance. Cambridge: Cambridge University Press.

North, Douglass, e Robert Thomas. 1973. The Rise of the Western World. Cambridge: Cambridge University Press.

Ostrom, Elinor. 2000. 'Collective Action and the Evolution of Social Norms'. Journal of Economic Perspectives 14 (3): pp. 137-58.

Sen, Amartya. 1967. 'Isolation, Assurance, and the Social Rate of Discount'. Quarterly Journal of Economics 81 (1): pp. 112-124.

Toniolo, Gianni. 2013. 'La crescita economica italiana, 1861-2011'. In L'Italia e l'economia mondiale dall'unità a oggi, a cura di Gianni Toniolo, pp. 5-51. Venezia: Marsilio.

Vecchi, Giovanni. 2017. Measuring Wellbeing: A History of Italian Living Standards. Oxford: Oxford University Press. 